\title{
CICADA: Cough in Children and Adults: Diagnosis and Assessment. Australian Cough Guidelines summary statement
}

\author{
Peter G Gibson, Anne B Chang, Nicholas J Glasgow, Peter W Holmes, Peter Katelaris, Andrew S Kemp, \\ Louis I Landau, Stuart Mazzone, Peter Newcombe, Peter Van Asperen and Anne E Vertigan
}

$\sim$ ough is a common and distressing symptom that results in significant health care costs from medical consultations and medication use. Non-specific cough suppressant therapy offers little benefit in managing persistent cough. Successful management requires a treatment program based on accurate diagnosis and understanding of the cough aetiology. CICADA (Cough in Children and Adults: Diagnosis and Assessment) is a clinical guideline for the assessment and management of persistent cough in children and adults. CICADA was developed by a multidisciplinary expert committee after a needs assessment by clinicians (summarised at http://www.lungfoundation.com.au/content/view/311/345). The guideline development process is further described in Box 1 .

\section{Definitions}

Cough is a reflex activity with elements of voluntary control. It forms part of the somatosensory system that involves visceral sensation, a reflex motor response and associated behavioural responses. ${ }^{4}$ Clinically useful definitions of cough are based on the duration of the symptom, whether it is associated with specific conditions recognised to cause cough, and its response (or lack of response) to treatment (Box 2). CICADA addresses protracted and chronic cough, and does not make recommendations for acute cough.

\section{Neurophysiology}

The cough reflex is dependent on sensory nerve fibres carried by the vagi that are responsive to chemical and mechanical stimuli. These fibres are integrated into a brainstem circuit that is responsible for generating the basic cough motor pattern that provides co-ordinated output via phrenic, intercostal, laryngeal and abdominal motor neurone pathways to the muscles involved in coughing. ${ }^{5}$

Cough is a protective reflex, and this function is relevant to cough that is secondary to the presence of a foreign body or mucus hypersecretion. Sensitisation of the cough reflex is a feature of patients with chronic cough and may occur via peripheral and/or central mechanisms. ${ }^{5}$ Both chemosensitive and mechanosensitive

\begin{tabular}{|c|c|}
\hline \multicolumn{2}{|c|}{ Abbreviations } \\
\hline ACE & Angiotensin-converting enzyme \\
\hline CICADA & Cough in Children and Adults: Diagnosis and Assessment \\
\hline CPAP & Continuous positive airway pressure \\
\hline CT & Computed tomography \\
\hline GORD & Gastro-oesophageal reflux disease \\
\hline OSA & Obstructive sleep apnoea \\
\hline PPI & Proton-pump inhibitor \\
\hline VCD & Vocal cord dysfunction \\
\hline
\end{tabular}

\section{ABSTRACT}

- Cough is a common and distressing symptom that results in significant health care costs from medical consultations and medication use.

- Cough is a reflex activity with elements of voluntary control that forms part of the somatosensory system involving visceral sensation, a reflex motor response and associated behavioural responses.

- At the initial assessment for chronic cough, the clinician should elicit any alarm symptoms that might indicate a serious underlying disease and identify whether there is a specific disease present that is associated with chronic cough.

- If the examination, chest x-ray and spirometry are normal, the most common diagnoses in ADULTS are asthma, rhinitis or gastro-oesophageal reflux disease (GORD). The most common diagnoses in CHILDREN are asthma and protracted bronchitis.

- Management of chronic cough involves addressing the common issues of environmental exposures and patient or parental concerns, then instituting specific therapy.

- In ADULTS, conditions that are associated with removable causes or respond well to specific treatment include protracted bacterial bronchitis, angiotensin-converting enzyme inhibitor use, asthma, GORD, obstructive sleep apnoea and eosinophilic bronchitis.

- In CHILDREN, diagnoses that are associated with removable causes or respond well to treatment are exposure to environmental tobacco smoke, protracted bronchitis, asthma, motor tic, habit and psychogenic cough.

- In ADULTS, refractory cough that persists after therapy is managed by empirical inhaled corticosteroid therapy and speech pathology techniques.

MJA 2010; 192: 265-271

cough fibres can be sensitised by inflammation in the airway, making them more responsive to a given stimulus (peripheral sensitisation). ${ }^{5}$ The activity of the brainstem circuitry can be tonically enhanced by persistent peripheral sensory nerve input, such that it becomes hyper-responsive to additional input from cough fibres (central sensitisation). Higher brain centres also modulate the basic cough reflex. ${ }^{5}$ Coughing can be voluntarily initiated and inhibited, ${ }^{6}$ is highly susceptible to placebo suppression, ${ }^{4,6}$ and is diminished during general anaesthesia or sleep. ${ }^{7}$ The urge-to-cough sensation, which may or may not precede a cough, may be related to subthreshold action potential levels or a different afferent pathway. ${ }^{8}$ 


\section{POSITION STATEMENT}

\section{Epidemiology}

In Australia, cough is one of the most common reasons for presenting for a medical consultation. ${ }^{9,10}$ Epidemiological studies identify a $5 \%-10 \%$ prevalence of chronic cough in both adults and children. ${ }^{9}$ Chronic cough has a negative impact on quality of life in adults $^{11,12}$ and in parents of children with chronic cough. ${ }^{9}$ In

\section{Process used in developing this document}

From May 2006 to April 2009, members of CICADA (Cough in Children and Adults: Diagnosis and Assessment) recurrently convened (by email, face-to-face and by teleconference) to discuss definitions followed by recommendations. The complete document will be available in 2010 on the Australian Lung Foundation website (http://www.lungfoundation.com.au). CICADA's recommendations are based on a successful problem-based clinical assessment method that is widely used in primary care. ${ }^{1}$ It uses the principles of evidence-based medicine and the Grading of Recommendations Assessment, Development and Evaluation (GRADE) approach ${ }^{2}$ to determine the strength of treatment recommendations made in guidelines: strong, weak, or "no specific recommendation".

Quoting from Guyatt et al in their article on the GRADE

classification, ${ }^{2}$ the implications of a strong recommendation are:

- For patients - most people in your situation would want the recommended course of action and only a small proportion would not; request discussion if the intervention is not offered;

- For clinicians - most patients should receive the recommended course of action;

- For policy makers - the recommendation can be adopted as a policy in most situations.

The implications of a weak recommendation are:

- For patients - most people in your situation would want the recommended course of action, but many would not;

- For clinicians - you should recognise that different choices will be appropriate for different patients and that you must help each patient to arrive at a management decision consistent with her or his values and preferences;

- For policy makers - policy making will require substantial debate and involvement of many stakeholders.

The implications of "no specific recommendation" are that the advantages and disadvantages are equivalent, the target population has not been identified, and/or there is insufficient evidence on which to formulate a recommendation. ${ }^{3}$

The CICADA multidisciplinary expert committee prepared the CICADA guideline. A subgroup ( $P G G$ and $A B C$ ) with expertise in evidence-based techniques and systematic reviews prepared a GRADE summary table for each recommendation using the nominal group technique. A PubMed search for the highest level of evidence relating to each recommendation was performed. The relevant citations and their abstracts were sent to CICADA members, who used the information to assess each recommendation.

The summary table assigned a grading to each of the domains of quality of evidence, balance between desirable and undesirable effects, values and preferences, and costs, which were assessed using the GRADE descriptors. ${ }^{2}$ The guideline and summary table were then reviewed by the CICADA multidisciplinary expert committee.

Consensus was achieved using a GRADE grid to record the views of committee members, and the strength of recommendations was assigned by formal voting rules. ${ }^{3}$ The final GRADE recommendations were based on these votes and considered cough in the context of the respective conditions (Box 6). adults, it may be associated with significant psychosocial disturbance, including anxiety and depression. ${ }^{13}$

\section{Diagnosis and assessment}

The initial assessment for protracted or chronic cough is intended to characterise the condition based on history, to elicit any alarm symptoms or findings that may indicate a serious underlying disease (Box 3), and to identify whether there is a specific disease present that is associated with chronic cough (in which case it is termed "specific cough").

\section{Specific cough}

Specific cough refers to a cough that occurs with a condition known to be associated with or cause chronic cough. Identification of the many different conditions that are associated with chronic cough forms the basis of specific treatment and further investigation. These conditions can be identified by a probability-based diagnostic approach (Box 4), by consideration of important conditions not to be missed (Box 5), and by reviewing cough pointers (eg, quality of cough, such as "brassy" cough, suggesting tracheomalacia; coexisting wheeze, suggesting asthma). ${ }^{14}$ Methods for systematic and objective cough assessment are also available, but at present they are mainly used in research settings or specialised cough clinics. ${ }^{15}$

\section{The GRADE system}

The Grading of Recommendations Assessment, Development and Evaluation (GRADE) system for classifying recommendations made in guidelines ${ }^{2}$ was developed by a multinational group of experts in research methods and evidence-based medicine. Based on an assessment of the strength of evidence, and possible adverse effects and costs of using the therapy, the CICADA panel used the GRADE system to determine the strength of cough treatment recommendations as strong, weak, or "no specific recommendation" (Box 1).

\section{Definitions of cough for clinical practice ${ }^{\star}$}

\begin{tabular}{|c|c|}
\hline Cough type & Definition \\
\hline Cough & $\begin{array}{l}\text { A forced expulsive manoeuvre, usually } \\
\text { against a closed glottis, that is associated } \\
\text { with a characteristic sound }\end{array}$ \\
\hline Acute cough & Cough lasting up to 2 weeks \\
\hline $\begin{array}{l}\text { Protracted acute } \\
\text { cough (in CHILDREN) }\end{array}$ & Cough lasting 2-4 weeks \\
\hline $\begin{array}{l}\text { Chronic cough (in } \\
\text { CHILDREN) }\end{array}$ & Cough lasting more than 4 weeks \\
\hline $\begin{array}{l}\text { Chronic persistent } \\
\text { cough (in ADULTS) }\end{array}$ & Cough lasting more than 8 weeks \\
\hline Specific cough & $\begin{array}{l}\text { Cough associated with a condition } \\
\text { recognised to cause cough }\end{array}$ \\
\hline Non-specific cough & $\begin{array}{l}\text { Cough without any specific disease } \\
\text { association }\end{array}$ \\
\hline Refractory cough & Cough that persists after therapy \\
\hline
\end{tabular}

* These definitions were adopted by the Cough in Children and Adults: Diagnosis and Assessment panel after consideration. The suggested times are indicative only. 


\section{POSITION STATEMENT}

\section{Management (Box 6)}

Management of protracted or chronic cough involves addressing the common issues of environmental exposures and patient or parental concerns, then instituting specific therapy. Management practices for infants and school-age children are differentiated from those for adults when considered appropriate. Although there are similarities between the two, there are also substantial differences. $^{16}$

Environmental exposures. Tobacco smoke exposure, both active and environmental, is a significant trigger for cough. Cessation of parental smoking can successfully reduce cough in children (GRADE: strong). ${ }^{17}$ Other potentially relevant exposures include certain forms of home heating, respiratory irritants (such as particulates) and proximity to a high level of road traffic. In ADULTS, the use of angiotensin-converting enzyme (ACE) inhibitors may be associated with persistent cough. ${ }^{18}$ Management consists of evaluation of the risks and benefits of ACE inhibitor therapy and cessation or initiation of alternative therapy, as appropriate.

Patient and parental concerns. Patients and their carers have significant concerns and fears in relation to the aetiology and outcome of a cough and the possible presence of a serious underlying disease. ${ }^{9}$ Providing information on the possible cough aetiology, time course for resolution of cough, and expected management may help reduce anxiety. ${ }^{19}$ Education is most effective when combined with a medical consultation. ${ }^{20}$ The provision of written information without health professional consultation has only modest benefits. ${ }^{21}$

\section{Alarm symptoms and findings in chronic cough} In ADULTS

- Haemoptysis

- Smoker with $>20$ pack-year smoking history

- Smoker over 45 years of age with a new cough, altered cough, or cough with voice disturbance

- Prominent dyspnoea, especially at rest or at night

- Substantial sputum production

- Hoarseness

- Systemic symptoms: fever, weight loss

- Complicated gastro-oesophageal reflux disease (GORD) associated with weight loss, anaemia, overt gastrointestinal bleeding (haematemesis or melaena), dysphagia, odynophagia, or empirical treatment failure for GORD

- Feeding difficulties (including choking or vomiting)

- Recurrent pneumonia

- Abnormal clinical respiratory examination

- Abnormal chest x-ray

In CHILDREN

- Prominent dyspnoea, especially at rest or at night

- Recurrent episodes of chronic, wet or productive cough

- Systemic symptoms: fever, weight loss, failure to thrive

- Feeding difficulties (including choking or vomiting)

- Recurrent pneumonia

- Stridor and other respiratory noises

- Abnormal clinical respiratory examination

- Abnormal chest x-ray

\section{Specific cough: lower airway disorders}

\section{Protracted bacterial bronchitis}

A diagnosis of protracted bacterial bronchitis is considered in patients with a protracted acute cough or a chronic cough that is wet, moist, or productive and occurs in the absence of other specific cough diagnoses or cough pointers. ${ }^{14}$ Chest x-ray and spirometry are usually normal. Medium-term antibiotic treatment (2-6 weeks) should lead to complete cough resolution (GRADE: strong). ${ }^{22}$ The diagnosis can only be definitive when patients become asymptomatic with treatment. ${ }^{23}$

\section{Probability-based diagnosis of chronic cough}

If examination, chest $x$-ray and spirometry are normal, the most common diagnoses or exposures associated with chronic cough are as follows:

\section{Adults}

Asthma

Rhinosinusitis

Gastro-oesophageal reflux disease

Children

Protracted bacterial bronchitis

Asthma (if other symptoms are present)

Exposures

Respiratory infection (post-infectious cough)

Tobacco smoke and other pollutants (active, environmental exposure)

\section{Remediable conditions and significant conditions not to be missed in people with chronic cough}

Conditions with specific remediable causes or usually good treatment response

- Protracted bacterial bronchitis

- Asthma

- Gastro-oesophageal reflux disease

- Obstructive sleep apnoea

In ADULTS

- Angiotensin-converting enzyme inhibitor use

- Eosinophilic bronchitis

In CHILDREN

Motor tic, habitual cough and psychogenic cough

Significant conditions not to be missed

In ADULTS

- Neoplasia of larynx, bronchus, or lung

- Parenchymal lung disease: chronic obstructive pulmonary disease, interstitial pulmonary fibrosis, bronchiectasis, sarcoidosis, chronic pulmonary collapse

- Infection: tuberculosis, lung abscess, pertussis

- Cardiovascular disease: left ventricular failure, pulmonary infarction, aortic aneurysm

In CHILDREN

- Congenital airway abnormalities

- Foreign body inhalation

- Parenchymal lung disease: cystic fibrosis, interstitial pulmonary fibrosis, bronchiectasis, sarcoidosis, chronic pulmonary collapse

- Infection: tuberculosis, lung abscess, pertussis 


\section{Recommendations for children and adults with cough*}

\section{In CHILDREN}

All cough types

Cough with allergic

rhinitis

Cough with obstructive sleep apnoea

Cough with asthma

Cough with protracted bacterial bronchitis

Cough with GORD

Non-specific or refractory cough
Recommended treatment/approach

Strength of recommendation ${ }^{\dagger}$

Cessation of parental smoking

According to current rhinitis management guidelines, involving topical nasal corticosteroids, antihistamines and allergen management

Tonsillectomy and adenoidectomy

Strong

Weak

Weak

According to current asthma management guidelines, involving education, selfmanagement, inhaled bronchodilators and inhaled corticosteroids

Medium-term antibiotic therapy (2-6 weeks) for protracted bacterial bronchitis

Strong

Strong

Empirical trial of high-dose PPI therapy (eg, any standard-dose PPI twice daily for 8-12 NSR weeks) if there is a reasonable suspicion that GORD may be contributing to chronic cough Laparoscopic fundoplication for chronic cough

Address patient/parental stress and concerns

Strong recommendation against surgery

Strong

Address exacerbating factors

Minimise use of medications other than demulcents such as honey (if no contraindications to its use exist)

Adopt counsel, watch, wait and review approach

Weak

Empirical trial of inhaled corticosteroid therapy

Empirical trial of PPIs

Speech pathology techniques designed to relieve glottal constriction during inspiration and to recognise and alter the response to precipitants

Antitussive therapy with narcotics

Strong

Strong

NSR

NSR

NSR

Strong recommendation against use

Weak

Strong

Weak corticosteroids, antibiotics and non-specific therapy

rhinosinusitis

According to current rhinitis management guidelines, involving topical nasal corticosteroids, antihistamines and allergen management

Cough with allergic

rhinitis

According to current rhinosinusitis management guidelines, involving topical nasal

Cough with vocal cord Medical management of comorbid conditions (eg, asthma, rhinosinusitis, GORD, dysfunction use of ACE inhibitors)

Speech pathology techniques designed to relieve glottal constriction during inspiration and to recognise and alter the response to precipitants

Cough with

obstructive sleep

Nasal continuous positive airway pressure

Strong

Strong

apnoea

Cough with asthma

According to current asthma management guidelines, involving education, selfmanagement, inhaled bronchodilators and inhaled corticosteroids

Cough with Inhaled corticosteroid therapy for 2-4 weeks

eosinophilic bronchitis

Cough with protracted bacterial bronchitis

Cough with GORD

Medium-term antibiotic therapy (2-6 weeks) for protracted bacterial bronchitis

Empirical trial of high-dose PPI therapy (eg, any standard-dose PPI twice daily for 8-12

Strong weeks) if there is a reasonable suspicion that GORD may be contributing to chronic cough Laparoscopic fundoplication for chronic cough

Non-specific and Address patient stress and concerns

NSR refractory cough

\section{Address exacerbating factors}

Empirical trial of inhaled corticosteroid therapy

Strong

Strong

Strong

Empirical trial of PPIs

Speech pathology techniques designed to relieve glottal constriction during inspiration and to recognise and alter the response to precipitants

Antitussive therapy with narcotics
Strong

Weak

Strong

Strong

Strong

NSR

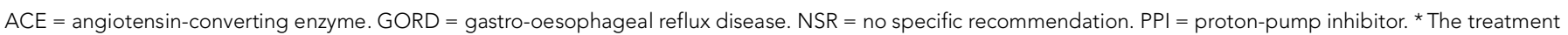
recommendation refers to the efficacy of treatment for cough occurring in association with the conditions specified. $†$ The strength of each recommendation was classified by the Cough in Children and Adults: Diagnosis and Assessment panel according to the GRADE system ${ }^{2}$ (see Box 1 for a description of the process used). 


\section{POSITION STATEMENT}

\section{Asthma}

Asthma is considered as a cause of chronic cough if the cough is episodic and associated with other features such as expiratory wheeze and/or exertional dyspnoea, or exhibits an obstructive ventilatory pattern on lung function testing (particularly if responsive to a bronchodilator). Other suggestive but less definitive features include the presence of exercise-induced cough or atopy. The diagnosis should be confirmed by spirometry with a positive bronchodilator response, or a positive bronchial provocation challenge. Treatment is according to current asthma management guidelines $^{24}$ and involves education, self-management and the use of inhaled bronchodilators and inhaled corticosteroids. Treatment is expected to be effective within 2-4 weeks (GRADE: strong).

In CHILDREN, chronic cough without the above features is seldom due to asthma, and inhaled corticosteroids are not indicated unless there are positive features to suggest asthma.

\section{Eosinophilic bronchitis in adults}

In ADULTS, eosinophilic bronchitis includes non-asthmatic eosinophilic bronchitis, eosinophilic asthma, cough-variant asthma and atopic cough. ${ }^{25}$ These conditions are characterised by chronic eosinophilic inflammation of the lower airway (eosinophilic bronchitis) that can be recognised from an induced sputum sample (eosinophils $>3 \%$ of non-squamous cells) or bronchoalveolar lavage (eosinophils $>2 \%$ ), and are suggested by an elevated fractional exhaled nitric oxide (FeNO) level (>47 ppb). ${ }^{26}$ Management in adults involves inhaled corticosteroid therapy, with a favourable response generally seen within 2-4 weeks (GRADE: strong). ${ }^{25}$ As access to eosinophil markers is limited, an empirical trial of inhaled corticosteroids can be considered where appropriate.

\section{Specific cough: upper airway disorders}

\section{Allergic rhinitis}

Allergic rhinitis can be diagnosed by eliciting signs and symptoms of nasal inflammation: nasal itching, nasal blockage, nasal discharge, conjunctivitis and nocturnal snoring. Relevant allergenic triggers can be identified by measuring allergen-specific IgE levels using skin prick testing or radioallergosorbent testing, with allergens limited by restriction to likely exposures or by exclusion of specific allergens. Indiscriminate use of a large number of allergens is not advised. The management of allergic rhinitis follows current evidence-based guidelines ${ }^{27}$ and involves treatment with a topical nasal steroid, with or without antihistamine (GRADE: weak). Allergen removal, allergen minimisation or immunotherapy can also be performed (GRADE: weak). Antihistamines have not been shown to be efficacious in children. ${ }^{28}$

The evidence that postnasal drip (discharge) is a significant cause of cough is tenuous. ${ }^{29}$ When postnasal drip or discharge is associated with cough, it is most likely a reflection of coexisting upper airway disease. ${ }^{30}$

\section{Chronic rhinosinusitis in adults}

In ADULTS, chronic rhinosinusitis as a cause of chronic cough is suggested by nasal or upper airway symptoms of more than 12 weeks' duration and at least two of the following symptoms or signs: anterior and/or posterior mucopurulent drainage, nasal obstruction, facial pressure, pain or fullness. Other suggestive symptoms include halitosis, hyposmia or anosmia, headaches, fatigue, dental pain, a sensation of ear pressure, the presence of inflammation on examination of a congested nose (discoloured mucus, oedema of the middle meatus) and/or a computed tomography (CT) scan showing evidence of rhinosinusitis. ${ }^{27}$

Investigation can involve a CT scan of the sinuses. This is indicated when the diagnosis is uncertain, the patient is not responding to medical treatment as expected, or surgery is planned or being considered. Bacterial culture of nasal swabs is indicated for patients who are not responding to medical therapy and to guide antibiotic selection. Allergy assessment can be helpful if there is associated allergic rhinitis. Management is according to current evidence-based guidelines ${ }^{27}$ and involves nasal saline irrigation, intranasal corticosteroid therapy for a minimum trial of 1 month, and oral antibiotic therapy for 3 weeks to 3 months for purulent chronic rhinosinusitis. Short-course prednisone treatment can be added for associated nasal polyposis (GRADE: strong).

\section{Obstructive sleep apnoea}

Obstructive sleep apnoea (OSA) has been increasingly recognised to be associated with chronic cough in adults and to a limited extent in children. ${ }^{31,32}$ OSA is suggested by a history of snoring associated with witnessed apnoeas, sleep disturbance or sweating at night; excessive daytime sleepiness; failure to thrive (in infants); obesity; enlarged tonsils; or nasal blockage. The diagnosis is confirmed by polysomnography. Treatment of OSA in ADULTS involves nasal continuous positive airway pressure (CPAP) (GRADE: strong). ${ }^{33}$ Treatment of OSA in CHILDREN involves nasal CPAP or tonsillectomy and adenoidectomy (GRADE: weak).

\section{Vocal cord dysfunction in adults}

In ADULTS, vocal cord dysfunction (VCD) is characterised by episodic and involuntary narrowing of the vocal cords during inspiration, leading to symptoms of inspiratory dyspnoea, stridor, cough, throat tightness and dysphonia. ${ }^{34}$ The underlying pathophysiology is thought to be extrathoracic airway hyper-responsiveness. Up to $50 \%$ of adults with VCD report significant persistent cough. VCD is diagnosed by observation of vocal fold narrowing (adduction) on laryngoscopy during a symptomatic episode, or a fall in inspiratory flow of more than $25 \%$ during airway provocation saline challenge. ${ }^{34}$ In adults with VCD, the treatment consists of medical management of comorbid conditions (eg, asthma, rhinosinusitis, gastro-oesophageal reflux disease [GORD] or ACE inhibitor use) (GRADE: weak), as well as speech pathology techniques designed to relieve glottal constriction during inspiration and to recognise and alter the response to precipitants (GRADE: strong). ${ }^{34,35}$

\section{Gastro-oesophageal reflux disease}

Chronic cough may represent an extraoesophageal manifestation of GORD, although there is controversy as to cause and effect. ${ }^{36}$ Cough associated with GORD may result from activation of the cough reflex by refluxate in the oesophagus, acid-induced laryngitis, laryngopharyngeal reflux, or pulmonary aspiration of refluxate. ${ }^{37}$ The patient should be assessed for heartburn and regurgitation and to determine whether GORD alarm symptoms are present (Box 3). If such symptoms are present, specialist review and endoscopy may be required. In the absence of alarm symptoms, investigation is of limited value.

In ADULTS, an empirical trial of high-dose proton-pump inhibitor (PPI) therapy (eg, any standard-dose PPI twice daily for 8-12 weeks) is indicated if there is a reasonable suspicion that GORD 


\section{POSITION STATEMENT}

may be contributing to chronic cough. Ineffective PPI therapy should be ceased (GRADE: strong). ${ }^{38}$ Non-acid reflux is a recently recognised condition that is associated with extra-oesophageal symptoms, including cough. The investigation and successful management of non-acid reflux requires further study. Laparoscopic fundoplication is reserved for the small minority of adults in whom the diagnosis of troublesome GORD is secure, symptoms fail to respond to PPI therapy, the patient is well informed about the therapeutic choices, and there is evidence of major complications of GORD or the risk of complications (eg, aspiration) (GRADE: no specific recommendation). ${ }^{39}$

No specific recommendation has been made by the committee for managing GORD in CHILDREN, but CICADA recommends against fundoplication for treating isolated cough in children (Box 6).

\section{Non-specific and refractory cough}

Non-specific cough is a chronic cough that is not associated with any of the cough pointers or cough-associated diagnoses. Investigations with chest $\mathrm{x}$-ray and spirometry are normal. Refractory cough is a cough that persists after therapy. Most patients with non-specific cough undergo spontaneous resolution or improvement.

In ADULTS, the management of non-specific cough and refractory cough includes addressing patient stress and concerns (GRADE: strong) and addressing exacerbating factors (GRADE: weak). Consider an empirical trial of therapy involving an inhaled corticosteroid (GRADE: strong), a PPI (GRADE: strong) and speech pathology (GRADE: strong).

In CHILDREN, adopt the approach of counsel, watch, wait and review $^{40}$ (GRADE: strong). Address parental stress and concerns (GRADE: strong). At each review in children and adults, look out for specific pointers. CICADA recommends against the use of narcotic cough suppressants in children (GRADE: strong).

\section{Acknowledgements}

We thank Karen Lather, Judy Henry and the staff at the Australian Lung Foundation for their assistance in preparing these guidelines. In addition to the authors, additional advice was provided by Dr Julie Marchant and Dr Chris Brown.

\section{Competing interests}

Peter Gibson and/or his institution have received grant funding for research projects from the National Health and Medical Research Council (NHMRC), Pharmaxis, and Novartis, and funding to lecture at educational meetings sponsored by the Thoracic Society of Australia and New Zealand, the American Thoracic Society, the European Respiratory Society, AstraZeneca, GlaxoSmithKline and Novartis. None of these activities were related to the content of this article. Peter Katelaris has been a speaker at educational forums sponsored by AstraZeneca, Janssen-Cilag and Nycomed, and has received unconditional funding support from Janssen-Cilag to present at a scientific meeting. Stuart Mazzone has received consultancy payments from GlaxoSmithKline for research activities unrelated to this article. Peter Van Asperen is a member of the Merck Sharp \& Dohme Paediatric Respiratory Advisory Board and receives occasional speaker fees associated with this membership.

\section{Author details}

Peter G Gibson, MB BS, FRACP, Respiratory Physician, ${ }^{1}$ and Honorary Professor ${ }^{2}$

Anne B Chang, FRACP, MPHTM, PhD, Paediatric Respiratory Physician, ${ }^{3}$ and Professor and Head of Respiratory Research ${ }^{4}$
Nicholas J Glasgow, FRACGP, MD, FAChPM, Dean of Medicine and Health Sciences ${ }^{5}$

Peter W Holmes, MB BS, FCCP, FRACP, Deputy Director, Respiratory and Sleep Medicine ${ }^{6}$

Peter Katelaris, MB BS, MD, FRACP, Clinical Associate Professor ${ }^{7}$

Andrew S Kemp, MB BS, FRACP, PhD, Professor of Paediatric Allergy and Clinical Immunology 8,9

Louis I Landau, AO, MD, FRACP, Director, Medical Workforce ${ }^{10}$

Stuart Mazzone, PhD, NHMRC RD Wright Research Fellow ${ }^{11}$

Peter Newcombe, DipT, BEd, PhD, Senior Lecturer ${ }^{12}$

Peter Van Asperen, MB BS, MD, FRACP, Macintosh Professor of

Paediatric Respiratory Medicine, ${ }^{9}$ and $\mathrm{Head}^{13}$

Anne E Vertigan, BAppSc(SpPath), MBA, PhD, Area Profession Director, Speech Pathology ${ }^{14}$

1 Department of Respiratory and Sleep Medicine, John Hunter

Hospital, Newcastle, NSW.

2 Woolcock Institute of Medical Research, Sydney, NSW.

3 Queensland Children's Respiratory Centre, Royal Children's Hospital, Brisbane, QLD.

4 Child Health Division, Menzies School of Health Research, Charles

Darwin University, Darwin, NT.

5 Australian National University, Canberra, ACT.

6 Monash Medical Centre, Melbourne, VIC.

7 Department of Gastroenterology, Concord Hospital, Sydney, NSW.

8 Department of Allergy and Immunology, The Children's Hospital at

Westmead, Sydney, NSW.

9 Discipline of Paediatrics and Child Health, University of Sydney,

Sydney, NSW.

10 Department of Health, Western Australia, Perth, WA.

11 School of Biomedical Sciences, University of Queensland, Brisbane, QLD.

12 School of Psychology, University of Queensland, Brisbane, QLD.

13 Department of Respiratory Medicine, The Children's Hospital at

Westmead, Sydney, NSW.

14 Hunter New England Health, Newcastle, NSW.

Correspondence: peter.gibson@hnehealth.nsw.gov.au

\section{References}

1 Murtagh J. Murtagh's general practice. 4th ed. Sydney: McGraw Hill, 2007.

2 Guyatt GH, Oxman AD, Kunz R, et al. Going from evidence to recommendations. BMJ 2008; 336: 1049-1051.

3 Jaeschke R, Guyatt GH, Dellinger P, et al. Use of GRADE grid to reach decisions on clinical practice guidelines when consensus is elusive. BMJ 2008; 337: a744

4 Widdicombe J, Eccles R, Fontana G. Supramedullary influences on cough. Respir Physiol Neurobiol 2006; 152: 320-328.

5 Canning BJ. Encoding of the cough reflex. Pulm Pharmacol Ther 2007; 20: 396-401.

6 Lee PC, Cotterill-Jones C, Eccles R. Voluntary control of cough. Pulm Pharmacol Ther 2002; 15: 317-320.

7 Nishino T, Tagaito Y, Isono S. Cough and other reflexes on irritation of airway mucosa in man. Pulm Pharmacol 1996; 9: 285-292.

8 Lee MG, Undem BJ. Basic mechanisms of cough: current understanding and remaining questions. Lung 2008; 186 Suppl 1: S10-S16.

9 Marchant JM, Newcombe PA, Juniper EF, et al. What is the burden of chronic cough for families? Chest 2008; 134: 303-309.

10 Britt H, Miller GC, Knox S, et al. General practice activity in Australia 2003-2004. Canberra: Australian Institute of Health and Welfare, 2004. (AlHW Cat. No. GEP 16; General Practice Series No. 16.)

11 Irwin RS, French CT, Fletcher KE. Quality of life in coughers. Pulm Pharmacol Ther 2002; 15: 283-286.

12 Birring SS, Matos S, Patel RB, et al. Cough frequency, cough sensitivity and health status in patients with chronic cough. Respir Med 2006; 100: 1105-1109.

13 McGarvey LP, Carton C, Gamble LA, et al. Prevalence of psychomorbidity among patients with chronic cough. Cough 2006; 2: 4 


\section{POSITION STATEMENT}

14 Chang AB, Landau LI, Van Asperen PP, et al. Cough in children: definitions and clinical evaluation. Position statement of the Thoracic Society of Australia and New Zealand. Med J Aust 2006; 184: 398-403.

15 Pavord ID, Chung KF. Management of chronic cough. Lancet 2008; 371: 1375-1384.

16 Chang AB. Cough: are children really different to adults? Cough 2005; $1: 7$.

17 Brand PL, Duiverman EJ. Coughing and wheezing children: improvement after parents stop smoking. Ned Tijdschr Geneeskd 1998; 142: 825-827.

18 Dicpinigaitis PV. Angiotensin-converting enzyme inhibitor-induced cough: ACCP Evidence-Based Clinical Practice Guidelines. Chest 2006; 129 (1 Suppl): 169S-173S.

19 Butler CC, Kinnersley P, Hood K, et al. Clinical course of acute infection of the upper respiratory tract in children: cohort study. BMJ 2003; 327: 10881089.

20 Fitzmaurice DA. Written information for treating minor illness. BMJ 2001; 322: 1193-1194.

21 Little P, Somerville J, Williamson I, et al. Randomised controlled trial of self management leaflets and booklets for minor illness provided by post. BMJ 2001; 322: 1214-1216, 1217.

22 Marchant JM, Morris P, Gaffney J, Chang AB. Antibiotics for prolonged moist cough in children. Cochrane Database Syst Rev 2005; (4): CD004822.

23 Chang $A B$, Redding GJ, Everard ML. Chronic wet cough: protracted bronchitis, chronic suppurative lung disease and bronchiectasis. Pediatr Pulmonol 2008; 43: 519-531.

24 National Asthma Council Australia. Asthma management handbook 2006. Melbourne: NACA, 2006.

25 Gibson PG, Fujimura M, Niimi A. Eosinophilic bronchitis: clinical manifestations and implications for treatment. Thorax 2002; 57: 178-182.

26 Taylor DR, Pijnenburg MW, Smith AD, de-Jongste JC. Exhaled nitric oxide measurements: clinical application and interpretation. Thorax 2006; 61: 817-827.

27 Wallace DV, Dykewicz MS, Bernstein DI, et al. The diagnosis and management of rhinitis: an updated practice parameter. J Allergy Clin Immunol 2008; 122 (2 Suppl): S1-S84.
28 Chang AB, Peake J, McElrea M. Anti-histamines for prolonged non-specific cough in children. Cochrane Database Syst Rev 2008; (2): CD005604.

29 Campanella SG, Asher MI. Current controversies: sinus disease and the lower airways. Pediatr Pulmonol 2001; 31: 165-172.

30 Kemp AS. Does post-nasal drip cause cough in childhood? Paediatr Respir Rev 2006; 7: 31-35.

31 Baik I, Kim J, Abbott RD, et al. Association of snoring with chronic bronchitis. Arch Intern Med 2008; 168: 167-173.

32 Birring SS, Ing AJ, Chan K, et al. Obstructive sleep apnoea: a cause of chronic cough. Cough 2007; $3: 7$.

33 Bonnet R, Jörres R, Downey R, et al. Intractable cough associated with the supine body position. Effective therapy with nasal CPAP. Chest 1995; 108 . 581-585.

34 Gibson PG, Vertigan AE. Speech pathology for chronic cough: a new approach. Pulm Pharmacol Ther 2009; 22: 159-162.

35 Vertigan AE, Theodoros DG, Gibson PG, Winkworth AL. Efficacy of speech pathology management for chronic cough: a randomised placebo controlled trial of treatment efficacy. Thorax 2006; 61: 1065-1069.

36 Vakil N, van Zanten SV, Kahrilas P, et al. The Montreal definition and classification of gastroesophageal reflux disease: a global evidence-based consensus. Am J Gastroenterol 2006; 101: 1900-1920.

37 Gatta L, Vaira D, Sorrenti G, et al. Meta-analysis: the efficacy of proton pump inhibitors for laryngeal symptoms attributed to gastro-oesophageal reflux disease. Aliment Pharmacol Ther 2007; 25: 385-392.

38 Chang AB, Lasserson TJ, Gaffney J, et al. Gastro-oesophageal reflux treatment for prolonged non-specific cough in children and adults. Cochrane Database Syst Rev 2006; (4): CD004823.

39 Swoger J, Ponsky J, Hicks DM, et al. Surgical fundoplication in laryngopharyngeal reflux unresponsive to aggressive acid suppression: a controlled study. Clin Gastroenterol Hepatol 2006; 4: 433-441.

40 Marchant JM, Masters IB, Taylor SM, et al. Evaluation and outcome of young children with chronic cough. Chest 2006; 129: 1132-1141.

(Received 24 Jun 2009, accepted 27 Oct 2009) 